\title{
Health-Related Quality of Life and Associated Factors Among People Living with HIV Infection at a Comprehensive Hospital in Ethiopia
}

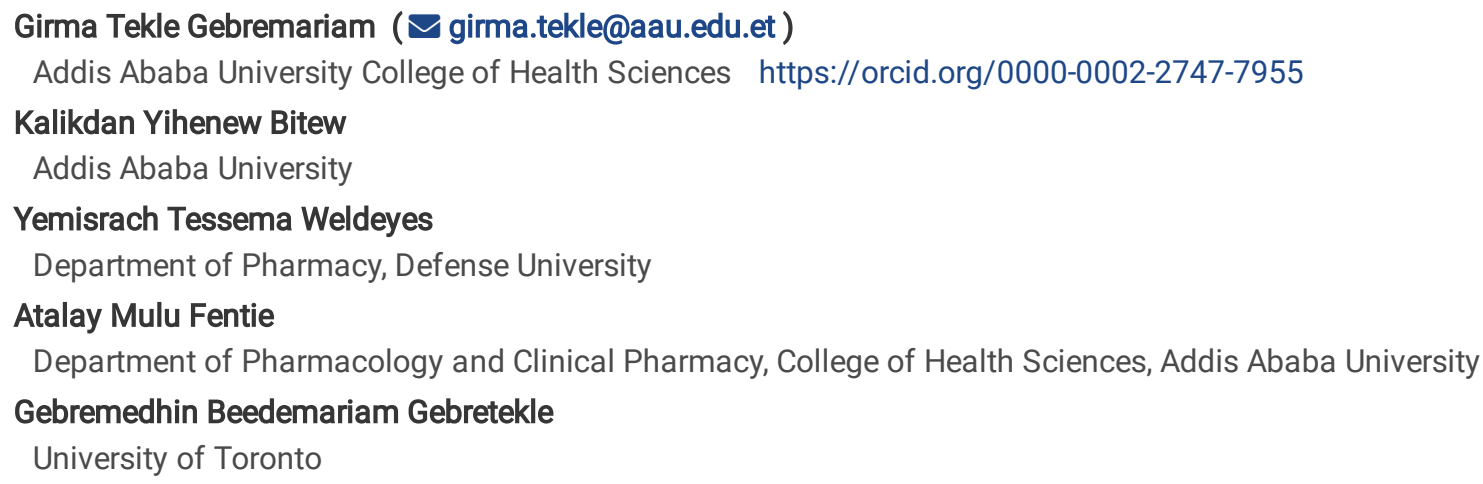

\section{Research}

Keywords: Health-related quality of life, HRQoL, HAT-QoL, People living with HIV, HAART

Posted Date: October 13th, 2020

DOI: https://doi.org/10.21203/rs.3.rs-89438/v1

License: (9) This work is licensed under a Creative Commons Attribution 4.0 International License. Read Full License 


\section{Abstract}

Background: Antiretroviral therapy (ART) has significantly reduced mortality and morbidity of people living with HIV/AIDS (PLWHA); however, the nature of infection and treatment associated with adverse drug reactions often adversely affect patient's health-related quality of life (HRQoL). The purpose of this study was to assess the HRQoL and its associated factors among PLWHA in Ethiopia.

Methods: A hospital-based cross-sectional study was conducted among patients taking HAART at Zewditu Memorial General Hospital in Addis Ababa. A face-to-face interview was performed using the HIV/AIDS targeted quality of Life (HAT-QoL) instrument. Patients who met eligibility criteria were approached consecutively while they came to the pharmacy for a medication refill. Descriptive statistics were used to present patient characteristics and HRQoL profiles. Independent t-test and one-way ANOVA were carried out to identify predictors for HRQoL. All statistical tests considered significant at $p$-value $<0.05$.

Results: A total of 300 participants were interviewed. The mean age of patients was $41.47 \pm 9.45$ years and the majority $(63.13 \%)$ were females. More than two-thirds $(69.7 \%)$ of patients were adherents to their medication. The highest HAT-QoL mean score was observed in the overall function domain $(84.96 \pm 19.87)$ followed by life satisfaction $(83.02 \pm 14.61)$ and physician trust $(80.53 \pm 20.79)$. Moderate mean scores were reported in the domains of health worries $(71.90 \pm 27.00)$, medication worries $(69.44 \pm 21.53)$ and disclosure worries (63.65 \pm 30.94$)$ had moderate mean scores, while HIV mastery (56.94 \pm 32.52$)$, financial worries $(48.81 \pm 44.64)$, and sexual function (12.57 \pm 45.56$)$ recorded lower mean scores. Being female, higher educational status, older age, low household income, taking prophylactic therapy, low CD4+ count, and non-adherence were significantly associated with HAT-QoL domains.

Conclusions: Overall, HIV/AIDS patients in Addis Ababa had an average level of HRQoL. This study demonstrated that several sociodemographic and clinical variables contribute to reduced HRQoL. Hence, healthcare providers should be paid attention to associated factors that contributed to reduced HRQoL.

\section{Introduction}

The HIV/AIDS pandemic continues to be an important public health problem worldwide in both high and low-income countries. Globally, nearly 38 million population have been living with HIV infection, of which 36.2 million were adults [1]. Although the burden of the epidemic lasts to differ considerably between countries and regions, Sub-Saharan Africa remains the most affected region accounting for $71 \%$ of the global burden [2, 3]. In 2019, 690,000 Ethiopians were living with HIV/AIDS: of the 650000 adults living with HIV, $63.08 \%$ were women [3, 4]. In the past two decades in Ethiopia, however, it has been observed a marked decline in HIV/AIDS morbidity and mortality (AIDS-related deaths decreased by 45\%, from 20000 deaths to 11000 deaths). The incidence of HIV infection has also reduced from 29000 to 23000 [3, 5].

The discovery of highly active antiretroviral therapy (HAART) improves patient survival and reduces the occurrence of HIV-related opportunistic infections [6]. However, drug-induced adverse events, the chronic and debilitating nature of HIV infection are adversely affecting patients' HRQoL [7-9]. Hence, monitoring the HRQoL of PLWHA has become an important indicator of the impact of disease and treatment outcomes $[6,10]$. Besides, measuring HRQoL can be used as an input for economic evaluation to generate evidence for priority setting and allocation of health care resources $[11,12]$.

HRQoL is increasingly recognized by clinicians and policymakers. Several studies have reported the impact of sociodemographic, behavioral, and disease-related characteristics on $\operatorname{HRQoL}[13,14]$. Indeed, co-morbidities, unemployment, and lack of social support were consistently reported to have adverse effects on HRQoL. In addition, significant associations of all HAT-QoL domains were observed with gender, age, residence, education, and marital status [15]. Other studies have also revealed that disease stage, viral loads, low CD4 count, and non-adherence to ART were important factors associated with poorer HRQoL [9, 16-18]. Furthermore, Sousa et al [19] reported that better HAT-QoL domain such as financial concern, concern about health, issues relating to medication, general activity, sexual activity, and satisfaction with life was associated with better living conditions. Therefore, the evidence illustrates that besides disease and HAART regimens, sociodemographic and clinical characteristics had a detrimental effect on the patients' HRQoL.

Various generic and disease-specific tools have been used to evaluate HRQoL in PLWHA [20-23]. The HIV specific instruments such as HATQoL are superior in capturing the impact of the disease and has good psychometric properties. Hence, this study employed the HAT-QoL measuring instrument to assess HRQoL and associated factors among PLWHA in a comprehensive hospital in Ethiopia [24]. Despite the availability of some studies on HRQoL of PLWHA using generic instruments in Ethiopia [25, 26], there is a paucity of HRQoL data using HATQoL in Ethiopia; hence, the study aimed to assess HRQoL and associated factors among patients living with HIV/AIDS attending at Zewditu memorial general hospital. 


\section{Methods \\ Study design and setting}

This was a cross-sectional study conducted among PLWHA receiving HAART at Zewditu Memorial General Hospital in Addis Ababa, Ethiopia, from March to June 2019. The hospital is the largest and pioneer HIV clinic in Ethiopia, with 18,000 HIV patients that receive comprehensive care and treatment. Three hundred PLWHA who met the eligibility criteria were consecutively approached for the interview using the HAT-QoL tool. Patients were considered eligible if they had confirmed diagnosis of HIV/AIDS infection and taking HAART for at least six months, were 18 years or older. While patients unwilling to participate, pregnant women, patients who had a cognitive or mental problem as per the suggestion of the physician, and patients taking HAART for less than 6 months were excluded from the study.

\section{Instruments}

The HAT-QoL is an HIV/AIDS specific quality of life measurement tool that consists of 42 items with 9 domains: overall function ( 6 items), life satisfaction (4 items), health worries ( 4 items), financial worries ( 3 items), medication worries ( 5 items), HIV mastery ( 2 items), disclosure worries (5 items), provider's trust (3 items), and sexual functions (2 items) (Supplementary file 1). Medication adherence was assessed using the Morisky green and Levine adherence scale questionnarie (MGL). Patient demographic characteristics (gender, age, marital status, occupation, level of education, living arrangement, substance use, and average monthly household income) were collected using structured questionnaire, whereas clinical characteristics (duration since diagnosis, CD4 Count, WHO staging, duration since HAART started, type of current regimen, opportunistic infections, comorbidity, adverse drug reactions, and types of prophylaxis therapy) were collected from patient medical records.

\section{Data collection procedures}

Trained pharmacy students collected the data through an interviewer-administered, face to face interview in a private/separate room near the pharmacy dispensing unit. Prior to the data collection, the purpose of the study and procedure of the task was explained for all consented participants. During the data collection process, data collectors clarify queries raised about the questionnaire for the patients. Data on patients' HRQoL, sociodemographic, as well as clinical characteristics, were collected while patients came for medications refill. The completeness and consistency of the collected data were assured at the end of the day.

\section{Ethical considerations}

The study was approved by Ethics Review Board of the School of Pharmacy, College of Health Sciences, Addis Ababa University (ERB/SOP/75/04/2019). Written informed consent was obtained from all participants before data collection and permission was taken from the hospital administration to conduct the study. We omitted personnel identifiers throughout the data collection, collected data were stored in a lockable cabinet, and access to data was restricted to the research team only and reported in aggregate.

\section{Statistical analysis}

Descriptive statistics (mean, standard deviation, and frequency) were used to summarize the socio-demographic and clinical characteristics as well as HRQoL profiles of participants. Coding was done in two ways, depending on the nature of the question. For positively worded questions, "All of the time" to "None of the time" responses were scored 5 to 1 respectively, while for the negatively worded ones, "All of the time" to "None of the time" responses were scored 1 to 5 . Participants needed to circle a number from 1 to 5 to indicate how often they have had such feelings in the past 4 weeks. Eight of the 42 items were negatively worded; these items were reverse scored for analysis, so that higher. Eight of the 42 items were negatively worded; these items were reverse scored for analysis so that higher scores indicated higher HRQoL in each domain. The final dimension score was a linear scale, where the mean score of items within each domain was used to calculate the domain scores and subsequently transformed to a 0 to 100 scale by using the questionnaire scoring guide, where 0 is the worst possible health state and 100 is the best possible health state[24]. Finally, the overall reported HRQoL of the patients were categorized based on the mean scores as "good health state > 75", "moderate health state; 50-74" and "low health state < 50 " [19, 27]. For the MGL scale, patients who reported 4 out of four categorized as "Good adherence" while one to three categorized as "Poor adherence". Independent t-test and one-way analysis of variance (ANOVA) were performed to identify factors associated with patients' HRQoL. Data were analyzed using SPSS version 23. All statistical tests are considered significant at $p$-value $<0.05$.

\section{Results}

\section{Patients characteristics}


A total of 300 PLWHA participated in the study; with a mean age of $41.47 \pm 9.45$ years. Most of the patients were women (65.3\%), married (29.3\%), and completed secondary school or above (64.9\%). About half (49.3\%) of the patients had $\mathrm{CD}_{4}$ count between $201-499$ cells/mm ${ }^{3}$; $152(50.7 \%)$ had lived with the disease for more than 10 years, 51 (17\%) had at least one opportunistic infections and 54 (18\%) had at least one comorbidity. The majority (73\%) of patients were asymptomatic HIV-positive and 145 (48.3\%) of the patients were on first-line regimen $(T D F+3 T C+E F V)$. Twenty-eight percent of patients took prophylaxis therapy for opportunistic infections, and $38 \%$ of patients' regimen has been changed, $19.3 \%$ due to adverse drug reaction accounts the largest. Two-thirds (69.7\%) of the PLWHA were adherent to their medications (Table 1). 
Table 1

Sociodemographic and clinical characteristics of people living with HIV/AIDS

\begin{tabular}{|c|c|}
\hline Variables & Number of patients (\%) \\
\hline \multicolumn{2}{|l|}{ Gender } \\
\hline Male & $104(34.7)$ \\
\hline Female & $196(65.3)$ \\
\hline \multicolumn{2}{|l|}{ Age categories } \\
\hline $18-34$ & $87(29.0)$ \\
\hline $35-49$ & $145(48.3)$ \\
\hline $50-64$ & $62(20.7)$ \\
\hline$\geq 65$ & $6(2.0)$ \\
\hline \multicolumn{2}{|l|}{ Marital status } \\
\hline Single & $73(24.3)$ \\
\hline Married & $88(29.3)$ \\
\hline Divorce & $75(25.1)$ \\
\hline Widowed & $64(21.3)$ \\
\hline \multicolumn{2}{|l|}{ Level of education } \\
\hline No formal education & $22(7.4)$ \\
\hline Primary & $83(27.7)$ \\
\hline Secondary & $103(34.3)$ \\
\hline Tertiary (diploma and above) & $92(30.6)$ \\
\hline \multicolumn{2}{|l|}{ Family monthly income (ETB) } \\
\hline$<500$ & $30(10.0)$ \\
\hline $500-1500$ & $58(19.3)$ \\
\hline $1501-3000$ & $58(19.3)$ \\
\hline $3001-5000$ & $35(11.7)$ \\
\hline$>5000$ & $61(20.3)$ \\
\hline Don't want to tell & $51(17.0)$ \\
\hline \multicolumn{2}{|l|}{ Living arrangement } \\
\hline Alone & $64(21.3)$ \\
\hline With family & $236(78.7)$ \\
\hline \multicolumn{2}{|l|}{ Substance use } \\
\hline Yes & $30(10.0)$ \\
\hline No & $270(90.0)$ \\
\hline \multicolumn{2}{|l|}{ Years since HIV/AIDS diagnosis } \\
\hline$<5$ years & $58(19.3)$ \\
\hline $5-10$ years & $90(30.0)$ \\
\hline > 10 years & $152(50.7)$ \\
\hline $1 S=31.32 E T B, I N H=$ Isoniazid & \\
\hline
\end{tabular}

Page 5/15 


\begin{tabular}{|c|c|}
\hline Variables & Number of patients (\%) \\
\hline \multicolumn{2}{|l|}{ CD 4 count (cells $/ \mathrm{mm}^{3}$ ) } \\
\hline$\geq 500$ & $99(33.0)$ \\
\hline $200-499$ & $148(49.3)$ \\
\hline$<200$ & $53(17.7)$ \\
\hline \multicolumn{2}{|l|}{ WHO staging } \\
\hline Stage I & $219(73.0)$ \\
\hline Stage II & $32(10.7)$ \\
\hline Stage III & $25(8.3)$ \\
\hline Stage IV & $24(8.0)$ \\
\hline \multicolumn{2}{|l|}{ Comorbidity } \\
\hline Yes & $54(18.0)$ \\
\hline No & $246(82.0)$ \\
\hline \multicolumn{2}{|c|}{ Presence of opportunistic infection } \\
\hline Yes & $51(17.0)$ \\
\hline No & $249(83.0)$ \\
\hline \multicolumn{2}{|c|}{ Types of opportunistic infection } \\
\hline Toxoplasmosis & $14(4.7)$ \\
\hline Fungal infections & $13(4.3)$ \\
\hline Tuberculosis & $9(3.0)$ \\
\hline Others & $15(5.0)$ \\
\hline \multicolumn{2}{|l|}{ Current HAART regimen } \\
\hline D4T-3TC-NVP & $35(11.7)$ \\
\hline D4T-3TC-EFV & $14(4.7)$ \\
\hline AZT-3TC-NVP & $29(9.7)$ \\
\hline AZT-3TC-EFV & $23(7.7)$ \\
\hline TDF-3TC-EFV & $145(48.3)$ \\
\hline TDF-3TC-NVP & $17(5.7)$ \\
\hline Others & $37(12.2)$ \\
\hline \multicolumn{2}{|l|}{ Prophylaxis therapy } \\
\hline Yes & $83(27.7)$ \\
\hline No & $217(72.3)$ \\
\hline \multicolumn{2}{|l|}{ Type of preventive therapy } \\
\hline Co-trimoxazole & $65(21.7)$ \\
\hline $\mathrm{INH}+$ Pyridoxine & $11(3.7)$ \\
\hline $\mathrm{INH}+$ Co-trimoxazole & $7(2.3)$ \\
\hline Initial regimen changed & \\
\hline
\end{tabular}

$1 \$=31.32 E T B, I N H=I$ soniazid 


\begin{tabular}{|cc|}
\hline Variables & Number of patients (\%) \\
\hline Yes & $112(37.3)$ \\
\hline No & $188(62.7)$ \\
\hline ADR & $47(42.0)$ \\
\hline Treatment failure & $28(25.0)$ \\
\hline Drug non-availability & $37(33.0)$ \\
\hline Adherence status & $209(69.7)$ \\
\hline Adherent & $91(30.3)$ \\
\hline Non-adherent & \\
\hline $1 S=31.32$ ETB, INH= Isoniazid & \\
\hline
\end{tabular}

\section{Health-related quality of Life of people with HIV infection}

The highest HRQoL score was observed in the domain of the overall function ( $84.96 \pm 19.87)$ followed by life satisfaction (83.02 \pm 14.61$)$ and physician trust $(80.53 \pm 20.79)$. The domains of health worries $(71.90 \pm 27.00)$, medication worries $(69.44 \pm 21.53)$, and disclosure worries $(63.65 \pm 30.94)$ had moderate mean scores, while the HIV mastery domain $(56.94 \pm 32.52)$, financial worries $(48.81 \pm 44.64)$, and sexual function (12.57 \pm 45.56$)$ reported lower mean scores (Fig. 1).

\section{Impact of sociodemographic characteristics on HRQoL}

The patient's gender was significantly associated with overall function, financial worries, HIV mastery, and disclosure worries domains. Health worries and sexual function domains were significantly associated with marital status, where the sexual function was better among married than single patients ( 49.1 vs 7.96; $p<0.001$ ). Educational status was significantly associated with five of the HAT-QoL domains: life satisfaction, health worries, financial worries, HIV mastery, and sexual function $(p<0.05)$. A statistically significant difference was observed in the mean scores for the health worries and disclosure worries among the different age groups. Participants who had low household income had more problems with financial, health, and medication worries. Furthermore, those patients who lived alone reported significantly high problems with sexual function ( 0.41 vs 16.0$)$ and disclosure worries than who live with family ( 53.1 vs 66.9$)$ (Table 2 ). 
Table 3

Impact of sociodemographic characteristics on HAT-QOL domains

\begin{tabular}{|c|c|c|c|c|c|c|c|c|c|}
\hline Variables & & LS* & HW & FW & MW & HM & & PT & SF \\
\hline \multicolumn{10}{|l|}{ Gender } \\
\hline Male & $\begin{array}{l}87.8 \pm \\
15.33\end{array}$ & $\begin{array}{l}82.9 \pm \\
10.81\end{array}$ & $\begin{array}{l}73.1 \pm \\
24.62\end{array}$ & $\begin{array}{l}63.1 \pm \\
43.46\end{array}$ & $\begin{array}{l}70.7 \pm \\
22.00\end{array}$ & $\begin{array}{l}61.9 \pm \\
32.94\end{array}$ & $\begin{array}{l}70.8 \pm \\
28.35\end{array}$ & $\begin{array}{l}80.6 \pm \\
20.92\end{array}$ & $\begin{array}{l}18.2 \\
\pm \\
45.60\end{array}$ \\
\hline Female & $\begin{array}{l}81.9 \pm \\
21.67\end{array}$ & $\begin{array}{l}84.6 \pm \\
16.27\end{array}$ & $\begin{array}{l}71.3 \pm \\
28.24\end{array}$ & $\begin{array}{l}41.2 \pm \\
43.49\end{array}$ & $\begin{array}{l}66.6 \pm \\
22.02\end{array}$ & $\begin{array}{l}54.3 \\
(32.06)\end{array}$ & $\begin{array}{l}59.9 \pm \\
31.66\end{array}$ & $\begin{array}{l}80.5 \pm \\
20.78\end{array}$ & $\begin{array}{l}9.6 \pm \\
45.38\end{array}$ \\
\hline$p$-value & & 0.348 & 0.568 & 0.000 & 0.127 & 0.040 & & 0.961 & 0.129 \\
\hline \multicolumn{10}{|l|}{ Marital status } \\
\hline Single & $\begin{array}{l}83.2 \pm \\
19.27\end{array}$ & $\begin{array}{l}82.4 \pm \\
10.65\end{array}$ & $\begin{array}{l}64.9 \pm \\
26.54\end{array}$ & $\begin{array}{l}54.3 \pm \\
42.38\end{array}$ & $\begin{array}{l}64.7 \pm \\
19.16\end{array}$ & $\begin{array}{l}58.1 \pm \\
33.82\end{array}$ & $\begin{array}{l}58.6 \\
(31.30)\end{array}$ & $\begin{array}{l}78.5 \pm \\
22.64\end{array}$ & $\begin{array}{l}7.96 \\
\pm \\
46.70\end{array}$ \\
\hline Married & $\begin{array}{l}86.2 \pm \\
16.84\end{array}$ & $\begin{array}{l}83.2 \pm \\
12.33\end{array}$ & $\begin{array}{l}78.3 \pm \\
23.67\end{array}$ & $\begin{array}{l}42.7 \pm \\
43.75\end{array}$ & $\begin{array}{l}67.5 \pm \\
24.11\end{array}$ & $\begin{array}{l}59.1 \pm \\
32.43\end{array}$ & $\begin{array}{l}67.8 \\
(32.00)\end{array}$ & $\begin{array}{l}77.7 \pm \\
21.72\end{array}$ & $\begin{array}{l}49.1 \\
\pm \\
34.89\end{array}$ \\
\hline Divorced & $\begin{array}{l}85.3 \pm \\
16.88\end{array}$ & $\begin{array}{l}83.4 \pm \\
11.18\end{array}$ & $\begin{array}{l}71.5 \pm \\
29.33\end{array}$ & $\begin{array}{l}55.2 \pm \\
45.96\end{array}$ & $\begin{array}{l}69.3 \pm \\
23.52\end{array}$ & $\begin{array}{l}57.7 \pm \\
31.77\end{array}$ & $\begin{array}{l}63.3 \pm \\
30.03\end{array}$ & $\begin{array}{l}83.0 \pm \\
19.05\end{array}$ & $\begin{array}{l}-7.77 \\
\pm \\
36.53\end{array}$ \\
\hline Widowed & $\begin{array}{l}80.1 \pm \\
26.45\end{array}$ & $\begin{array}{l}87.8 \pm \\
22.55\end{array}$ & $\begin{array}{l}68.5 \pm \\
27.73\end{array}$ & $\begin{array}{l}43.4 \pm \\
45.96\end{array}$ & $\begin{array}{l}71.0 \pm \\
20.36\end{array}$ & $\begin{array}{l}51.8 \pm \\
32.24\end{array}$ & $\begin{array}{l}63.0 \pm \\
29.93\end{array}$ & $\begin{array}{l}83.9 \pm \\
18.77\end{array}$ & $\begin{array}{l}-10.2 \\
\frac{ \pm}{34.57}\end{array}$ \\
\hline$p$-value & 0.268 & 0.132 & 0.028 & 0.153 & 0.381 & 0.554 & 0.303 & 0.168 & 0.000 \\
\hline \multicolumn{10}{|l|}{ Educational status } \\
\hline $\begin{array}{l}\text { Informal } \\
\text { education }\end{array}$ & $\begin{array}{l}88.4 \pm \\
9.11\end{array}$ & $\begin{array}{l}85.9 \pm \\
8.18\end{array}$ & $\begin{array}{l}73.9 \pm \\
8.84\end{array}$ & $\begin{array}{l}90.4 \pm \\
12.94\end{array}$ & $\begin{array}{l}82.0 \pm \\
12.69\end{array}$ & $\begin{array}{l}57.3 \pm \\
29.36\end{array}$ & $\begin{array}{l}74.4 \pm \\
18.79\end{array}$ & $\begin{array}{l}91.7 \pm \\
15.43\end{array}$ & $\begin{array}{l}-25.0 \\
\pm \\
0.00\end{array}$ \\
\hline $\begin{array}{l}\text { Primary } \\
\text { school }\end{array}$ & $\begin{array}{l}81.6 \pm \\
19.65\end{array}$ & $\begin{array}{l}83.1 \pm \\
11.5\end{array}$ & $\begin{array}{l}68.4 \pm \\
29.70\end{array}$ & $\begin{array}{l}29.5 \pm \\
41.04\end{array}$ & $\begin{array}{l}66.4 \pm \\
24.37\end{array}$ & $48.5 \pm 31.0$ & $\begin{array}{l}62.9 \pm \\
30.57\end{array}$ & $\begin{array}{l}78.9 \pm \\
18.7\end{array}$ & $\begin{array}{l}20.9 \\
\pm \\
45.15\end{array}$ \\
\hline $\begin{array}{l}\text { Secondary } \\
\text { school }\end{array}$ & $\begin{array}{l}86.2 \pm \\
16.63\end{array}$ & $\begin{array}{l}82.9 \pm \\
11.94\end{array}$ & $\begin{array}{l}70.4 \pm \\
26.00\end{array}$ & $\begin{array}{l}53.4 \pm \\
43.22\end{array}$ & $\begin{array}{l}66.6 \pm \\
22.69\end{array}$ & $\begin{array}{l}56.8 \pm \\
32.65\end{array}$ & $\begin{array}{l}61.7 \pm \\
31.26\end{array}$ & $\begin{array}{l}81.2 \pm \\
20.97\end{array}$ & $\begin{array}{l}8.8 \pm \\
45.44\end{array}$ \\
\hline $\begin{array}{l}\text { Higher } \\
\text { education }\end{array}$ & $\begin{array}{l}82.5 \pm \\
23.60\end{array}$ & $\begin{array}{l}83.7 \pm \\
11.11\end{array}$ & $\begin{array}{l}96.0 \pm \\
25.11\end{array}$ & $\begin{array}{l}66.9 \pm \\
41.60\end{array}$ & $\begin{array}{l}69.0 \pm \\
20.47\end{array}$ & $\begin{array}{l}64.0 \pm \\
32.91\end{array}$ & $\begin{array}{l}62.7 \pm \\
32.80\end{array}$ & $\begin{array}{l}79.6 \pm \\
23.2\end{array}$ & $\begin{array}{l}13.5 \\
\pm \\
45.56\end{array}$ \\
\hline$p$-value & 0.316 & 0.006 & 0.027 & 0.000 & 0.281 & 0.030 & 0.164 & 0.443 & 0.045 \\
\hline \multicolumn{10}{|l|}{ Age categories } \\
\hline $18-34$ & $\begin{array}{l}84.8 \pm \\
19.14\end{array}$ & $\begin{array}{l}81.8 \pm \\
11.92\end{array}$ & $\begin{array}{l}67.4 \pm \\
29.13\end{array}$ & $\begin{array}{l}51.1 \pm \\
43.05\end{array}$ & $\begin{array}{l}67.2 \pm \\
21.63\end{array}$ & $\begin{array}{l}53.3 \pm \\
31.14\end{array}$ & $\begin{array}{l}85.8 \pm \\
33.53\end{array}$ & $\begin{array}{l}81.5 \pm \\
20.94\end{array}$ & $\begin{array}{l}17.9 \\
\pm \\
47.93\end{array}$ \\
\hline $35-49$ & $\begin{array}{l}83.6 \pm \\
20.17\end{array}$ & $\begin{array}{l}84.9 \pm \\
17.24\end{array}$ & $\begin{array}{l}69.9 \pm \\
27.37\end{array}$ & $\begin{array}{l}46.4 \pm \\
45.28\end{array}$ & $\begin{array}{l}68.0 \pm \\
22.78\end{array}$ & $\begin{array}{l}56.8 \pm \\
34.36\end{array}$ & $\begin{array}{l}64.5 \pm \\
30.51\end{array}$ & $\begin{array}{l}79.4 \pm \\
21.36\end{array}$ & $\begin{array}{l}8.20 \\
\pm \\
43.67\end{array}$ \\
\hline $50-64$ & $\begin{array}{l}83.9 \pm \\
19.48\end{array}$ & $\begin{array}{l}84.6 \pm \\
11.16\end{array}$ & $\begin{array}{l}82.7 \pm \\
18.88\end{array}$ & $\begin{array}{l}51.2 \pm \\
45.16\end{array}$ & $\begin{array}{l}69.6 \pm \\
21.90\end{array}$ & $\begin{array}{l}62.5 \pm \\
29.71\end{array}$ & $\begin{array}{l}70.2 \pm \\
26.90\end{array}$ & $\begin{array}{l}82.1 \pm \\
19.84\end{array}$ & $\begin{array}{l}14.6 \\
\pm \\
45.81\end{array}$ \\
\hline$>65$ & $\begin{array}{l}80.9 \pm \\
30.86\end{array}$ & $\begin{array}{l}88.5 \pm \\
10.01\end{array}$ & $\begin{array}{l}72.5 \pm \\
34.31\end{array}$ & $\begin{array}{l}50.0 \pm \\
54.77\end{array}$ & $\begin{array}{l}62.5 \pm \\
13.69\end{array}$ & $\begin{array}{l}56.9 \pm \\
34.33\end{array}$ & $\begin{array}{l}60.0 \pm \\
33.47\end{array}$ & $\begin{array}{l}76.4 \pm \\
16.17\end{array}$ & $\begin{array}{l}20.8 \\
\frac{ \pm}{5} 4.45\end{array}$ \\
\hline P-value & 0.955 & 0.343 & 0.004 & 0.843 & 0.855 & 0.404 & 0.016 & 0.753 & 0.438 \\
\hline
\end{tabular}




\begin{tabular}{|c|c|c|c|c|c|c|c|c|c|}
\hline Variables OF* & & LS* & HW & FW & MW & HM & DW & PT & SF \\
\hline \multicolumn{10}{|c|}{ Household income (ETB)** } \\
\hline$<500$ & $\begin{array}{l}78.01 \pm \\
21.8\end{array}$ & $\begin{array}{l}83.3 \pm \\
17.67\end{array}$ & $\begin{array}{l}66.8 \pm \\
12.34\end{array}$ & $\begin{array}{l}42.5 \pm \\
43.53\end{array}$ & $\begin{array}{l}70.6 \pm \\
20.18\end{array}$ & $\begin{array}{l}60.6 \pm \\
21.90\end{array}$ & $\begin{array}{l}71.8 \pm \\
23.83\end{array}$ & $\begin{array}{l}75.6 \pm \\
16.51\end{array}$ & $\begin{array}{l}4.88 \\
\pm \\
40.06\end{array}$ \\
\hline $501-1500$ & $\begin{array}{l}81.9 \pm \\
19.45\end{array}$ & $\begin{array}{l}85.1 \pm \\
10.63\end{array}$ & $\begin{array}{l}76.3 \pm \\
24.35\end{array}$ & $\begin{array}{l}45.0 \pm \\
44.01\end{array}$ & $\begin{array}{l}74.9 \pm \\
20.20\end{array}$ & $\begin{array}{l}56.7 \pm \\
32.75\end{array}$ & $\begin{array}{l}57.6 \pm \\
29.10\end{array}$ & $\begin{array}{l}80.6 \pm \\
19.09\end{array}$ & $\begin{array}{l}9.52 \\
\pm \\
45.07\end{array}$ \\
\hline $1501-3000$ & $\begin{array}{l}78.0 \pm \\
21.18\end{array}$ & $\begin{array}{l}84.9 \pm \\
24.40\end{array}$ & $\begin{array}{l}71.6 \pm \\
26.92\end{array}$ & $\begin{array}{l}44.9 \pm \\
44.01\end{array}$ & $\begin{array}{l}62.5 \pm \\
21.63\end{array}$ & $\begin{array}{l}55.8 \pm \\
29.40\end{array}$ & $\begin{array}{l}62.4 \pm \\
32.37\end{array}$ & $\begin{array}{l}73.1 \pm \\
23.78\end{array}$ & $\begin{array}{l}17.4 \\
\pm \\
44.25\end{array}$ \\
\hline $3001-5000$ & $\begin{array}{l}81.7 \pm \\
23.28\end{array}$ & $\begin{array}{l}84.1 \\
11.05\end{array}$ & $\begin{array}{l}65.4 \pm \\
30.28\end{array}$ & $\begin{array}{l}33.8 \pm \\
41.46\end{array}$ & $\begin{array}{l}58.9 \pm \\
22.34\end{array}$ & $\begin{array}{l}53.3 \pm \\
34.69\end{array}$ & $\begin{array}{l}59.6 \pm \\
35.90\end{array}$ & $\begin{array}{l}82.4 \pm \\
17.36\end{array}$ & $\begin{array}{l}14.5 \\
\pm \\
48.99\end{array}$ \\
\hline$>5000$ & $\begin{array}{l}88.4 \pm \\
15.78\end{array}$ & $\begin{array}{l}82.7 \pm \\
10.57\end{array}$ & $\begin{array}{l}64.7 \pm \\
30.12\end{array}$ & $\begin{array}{l}45.2 \pm \\
44.52\end{array}$ & $\begin{array}{l}69.9 \pm \\
21.98\end{array}$ & $\begin{array}{l}61.9 \pm \\
31.71\end{array}$ & $\begin{array}{l}68.6 \pm \\
30.62\end{array}$ & $\begin{array}{l}78.9 \pm \\
22.44\end{array}$ & $\begin{array}{l}14.7 \\
\pm \\
45.68\end{array}$ \\
\hline $\begin{array}{l}\text { Don't want } \\
\text { to tell }\end{array}$ & $\begin{array}{l}88.2 \pm \\
19.39\end{array}$ & $\begin{array}{l}82.3 \pm \\
12.45\end{array}$ & $\begin{array}{l}75.9 \pm \\
20.52\end{array}$ & $\begin{array}{l}71.3 \pm \\
39.69\end{array}$ & $\begin{array}{l}68.8 \pm \\
21.16\end{array}$ & $\begin{array}{l}54.7 \pm \\
30.97\end{array}$ & $\begin{array}{l}64.4 \pm \\
30.68\end{array}$ & $\begin{array}{l}86.3 \pm \\
19.90\end{array}$ & $\begin{array}{l}12.6 \\
\pm \\
49.3\end{array}$ \\
\hline$p$-value & 0.056 & 0.902 & 0.048 & 0.000 & 0.005 & 0.763 & 0.246 & 0.030 & 0.676 \\
\hline \multicolumn{10}{|c|}{ Living arrangement } \\
\hline Alone & $\begin{array}{l}84.8 \pm \\
18.52\end{array}$ & $\begin{array}{l}81.5 \pm \\
11.39\end{array}$ & $\begin{array}{l}71.7 \pm \\
27.85\end{array}$ & $\begin{array}{l}47.1 \pm \\
47.52\end{array}$ & $\begin{array}{l}63.5 \pm \\
22.91\end{array}$ & $\begin{array}{l}56.8 \pm \\
31.35\end{array}$ & $\begin{array}{l}53.1 \pm \\
34.01\end{array}$ & $\begin{array}{l}81.6 \pm \\
20.58\end{array}$ & $\begin{array}{l}0.41 \\
\pm \\
44.25\end{array}$ \\
\hline With family & $\begin{array}{l}83.8 \pm \\
20.23\end{array}$ & $\begin{array}{l}84.7 \pm \\
15.35\end{array}$ & $\begin{array}{l}72.0 \pm \\
26.87\end{array}$ & $\begin{array}{l}49.4 \pm \\
43.98\end{array}$ & $\begin{array}{l}69.3 \pm \\
21.74\end{array}$ & $\begin{array}{l}57.1 \pm \\
32.93\end{array}$ & $\begin{array}{l}66.9 \pm \\
29.43\end{array}$ & $\begin{array}{l}80.3 \pm \\
20.90\end{array}$ & $\begin{array}{l}16.0 \\
\pm \\
34.25\end{array}$ \\
\hline$p$-value & 0.379 & 0.287 & 0.820 & 0.815 & 0.152 & 0.767 & 0.003 & 0.720 & 0.042 \\
\hline
\end{tabular}

\section{Impact of clinical characteristics on HRQoL}

Living with the disease for a longer duration was significantly associated with lower mean scores in the HAT-QoL domain of provider trust $(p=0.018)$, life satisfaction $(p=0.036)$, and health worries $(p=0.016)$. Those patients with a CD4 count of fewer than 200 cells $/ \mathrm{mm}^{3}$ and WHO stage IV reported significantly low mean scores in health worries. The result of this study demonstrated that PLWHA who took cotrimoxazole and Isoniazid preventive therapy had more health worries and financial worries than their counterpart. Life satisfaction was negatively associated with the presence of comorbidity, where patients with comorbidities had significantly lower life satisfaction mean score compared to patients who had no comorbidities (68.5 vs $87.3 ; p=0.030)$. The mean score of medication worries domain was significantly lower in patients who experienced HAART related adverse reactions than not experienced adverse reactions (21.3 vs 53.8; $p=$ 0.021). Compared to patients who were non-adherent to their medication, participants who were adherent had better overall function (85.4 vs $80.8 ; p=0.036$ ), and HIV mastery (58.2 vs $54.0 ; p=0.030$ ) (Table 3 ). 
Table 4

Impact of disease-related factors on HAT-QOL

\begin{tabular}{|c|c|c|c|c|c|c|c|c|c|}
\hline Variables & OF* & LS* & $H W *$ & FW* & $M W *$ & $\mathrm{HM}^{*}$ & $D W *$ & PT* & SF* \\
\hline \multicolumn{10}{|l|}{ Years since diagnosis } \\
\hline$<5$ years & $\begin{array}{l}86.8 \pm \\
22.1\end{array}$ & $\begin{array}{l}84.3 \pm \\
10.6\end{array}$ & $\begin{array}{l}69.9 \pm \\
30.1\end{array}$ & $\begin{array}{l}50.5 \pm \\
19.5\end{array}$ & $\begin{array}{l}45.3 \pm \\
5.45\end{array}$ & $\begin{array}{l}54.2 \pm \\
33.1\end{array}$ & $\begin{array}{l}58.8 \pm \\
31.9\end{array}$ & $\begin{array}{l}81.5 \pm \\
18.2\end{array}$ & $\begin{array}{l}10.3 \pm \\
46.0\end{array}$ \\
\hline $5-10$ years & $\begin{array}{l}83.2 \pm \\
19.5\end{array}$ & $\begin{array}{l}86.9 \pm \\
19.5\end{array}$ & $\begin{array}{l}71.2 \pm \\
26.1\end{array}$ & $\begin{array}{l}48.6 \pm \\
30.6\end{array}$ & $\begin{array}{l}45.0 \pm \\
4.69\end{array}$ & $\begin{array}{l}57.2 \pm \\
32.1\end{array}$ & $\begin{array}{l}64.5 \pm \\
30.7\end{array}$ & $\begin{array}{l}82.8 \pm \\
18.8\end{array}$ & $\begin{array}{l}7.50 \pm \\
46.3\end{array}$ \\
\hline$>10$ years & $\begin{array}{l}80.0 \pm \\
18.8\end{array}$ & $\begin{array}{l}81.9 \pm \\
12.0\end{array}$ & $\begin{array}{l}73.3 \pm \\
26.1\end{array}$ & $\begin{array}{l}48.1 \pm \\
15.5\end{array}$ & $\begin{array}{l}44.4 \pm \\
3.77\end{array}$ & $\begin{array}{l}58.1 \pm \\
32.7\end{array}$ & $\begin{array}{l}65.5 \pm \\
30.6\end{array}$ & $\begin{array}{l}88.5 \pm \\
23.1\end{array}$ & $\begin{array}{l}17.2 \pm \\
43.9\end{array}$ \\
\hline$p$-value & 0.185 & 0.036 & 0.016 & 0.935 & 0.865 & 0.719 & 0.331 & 0.018 & 0.284 \\
\hline \multicolumn{10}{|l|}{ WHO staging } \\
\hline Stage I & $\begin{array}{l}83.9 \pm \\
20.07\end{array}$ & $\begin{array}{l}84.2 \pm \\
15.5\end{array}$ & $\begin{array}{l}78.7 \pm \\
27.1\end{array}$ & $\begin{array}{l}49.6 \pm \\
45.0\end{array}$ & $\begin{array}{l}68.3 \pm \\
21.7\end{array}$ & $\begin{array}{l}32.52 \\
\pm .12\end{array}$ & $\begin{array}{l}63.0 \pm \\
30.6\end{array}$ & $\begin{array}{l}79.9 \pm \\
21.2\end{array}$ & $\begin{array}{l}12.1 \pm \\
45.3\end{array}$ \\
\hline Stage II & $\begin{array}{l}86.1 \pm \\
23.5\end{array}$ & $\begin{array}{l}84.2 \pm \\
7.69\end{array}$ & $\begin{array}{l}73.6 \pm \\
29.8\end{array}$ & $\begin{array}{l}58.3 \pm \\
42.2\end{array}$ & $\begin{array}{l}71.32 \pm \\
4.4\end{array}$ & $\begin{array}{l}30.0 \pm \\
7.30\end{array}$ & $\begin{array}{l}59.7 \pm \\
36.5\end{array}$ & $\begin{array}{l}80.4 \pm \\
23.7\end{array}$ & $\begin{array}{l}26.6 \pm \\
51.5\end{array}$ \\
\hline Stage III & $\begin{array}{l}85.8 \pm \\
16.7\end{array}$ & $\begin{array}{l}83.1 \pm \\
12.0\end{array}$ & $\begin{array}{l}76.0 \pm \\
26.0\end{array}$ & $\begin{array}{l}32.5 \pm \\
41.1\end{array}$ & $\begin{array}{l}64.0 \pm \\
22.8\end{array}$ & $\begin{array}{l}33.1 \pm \\
6.14\end{array}$ & $\begin{array}{l}68.8 \pm \\
27.2\end{array}$ & $\begin{array}{l}87.4 \pm \\
16.3\end{array}$ & $\begin{array}{l}15.2 \pm \\
45.6\end{array}$ \\
\hline Stage IV & $\begin{array}{l}79.8 \pm \\
19.2\end{array}$ & $\begin{array}{l}83.6 \pm \\
11.6\end{array}$ & $\begin{array}{l}65.6 \pm \\
26.0\end{array}$ & $\begin{array}{l}55.44 \pm \\
4.1\end{array}$ & $\begin{array}{l}67.5 \pm \\
23.7\end{array}$ & $\begin{array}{l}35.1 \pm \\
7.98\end{array}$ & $\begin{array}{l}67.3 \pm \\
33.3\end{array}$ & $\begin{array}{l}78.3 \pm \\
18.8\end{array}$ & $\begin{array}{l}2.9 \pm \\
43.5\end{array}$ \\
\hline$p$-value & 0.724 & 0.984 & 0.002 & 0.160 & 0.710 & 0.778 & 0.695 & 0.312 & 0.474 \\
\hline \multicolumn{10}{|c|}{ CD 4 Count (cells $/ \mathrm{mm}^{3}$ ) } \\
\hline$>500$ & $\begin{array}{l}83.9 \pm \\
20.63\end{array}$ & $\begin{array}{l}84.1 \pm \\
19.6\end{array}$ & $\begin{array}{l}78.8 \pm \\
26.1\end{array}$ & $\begin{array}{l}50.4 \pm \\
45.6\end{array}$ & $\begin{array}{l}68.2 \pm \\
20.3\end{array}$ & $\begin{array}{l}59.5 \pm \\
31.9\end{array}$ & $\begin{array}{l}64.3 \pm \\
31.8\end{array}$ & $\begin{array}{l}81.0 \pm \\
19.8\end{array}$ & $\begin{array}{l}11.7 \pm \\
45.4\end{array}$ \\
\hline $201-499$ & $\begin{array}{l}84.2 \pm \\
19.72\end{array}$ & $\begin{array}{l}84.6 \pm \\
11.3\end{array}$ & $\begin{array}{l}71.7 \pm \\
27.1\end{array}$ & $\begin{array}{l}50.1 \pm \\
44.2\end{array}$ & $\begin{array}{l}69.4 \pm \\
23.1\end{array}$ & $\begin{array}{l}57.4 \pm \\
33.1\end{array}$ & $\begin{array}{l}64.0 \pm \\
30.6\end{array}$ & $\begin{array}{l}79.3 \pm \\
21.8\end{array}$ & $\begin{array}{l}14.4 \pm \\
46.8\end{array}$ \\
\hline$<200$ & $\begin{array}{l}83.3 \pm \\
19.19\end{array}$ & $\begin{array}{l}83.9 \pm \\
10.9\end{array}$ & $\begin{array}{l}66.6 \pm \\
28.4\end{array}$ & $\begin{array}{l}42.6 \pm \\
44.2\end{array}$ & $\begin{array}{l}64.0 \pm \\
22.4\end{array}$ & $\begin{array}{l}51.0 \pm \\
31.9\end{array}$ & $\begin{array}{l}61.6 \pm \\
30.6\end{array}$ & $\begin{array}{l}82.6 \pm \\
19.7\end{array}$ & $\begin{array}{l}9.5 \pm \\
43.2\end{array}$ \\
\hline$p$-value & 0.095 & 0.861 & 0.028 & 0.521 & 0.014 & 0.276 & 0.864 & 0.576 & 0.771 \\
\hline \multicolumn{10}{|l|}{ Prophylaxis therapy } \\
\hline Yes & $\begin{array}{l}83.3 \pm \\
17.98\end{array}$ & $\begin{array}{l}84.3 \pm \\
10.2\end{array}$ & $\begin{array}{l}65.3 \pm \\
30.1\end{array}$ & $\begin{array}{l}40.8 \pm \\
44.8\end{array}$ & $\begin{array}{l}65.8 \pm \\
21.0\end{array}$ & $\begin{array}{l}54.2 \pm \\
32.5\end{array}$ & $\begin{array}{l}58.9 \pm \\
32.6\end{array}$ & $\begin{array}{l}81.4 \pm \\
20.3\end{array}$ & $\begin{array}{l}4.8 \pm \\
40.2\end{array}$ \\
\hline No & $\begin{array}{l}84.2 \pm \\
20.54\end{array}$ & $\begin{array}{l}83.9 \pm \\
15.9\end{array}$ & $\begin{array}{l}74.3 \pm \\
25.5\end{array}$ & $\begin{array}{l}51.7 \pm \\
44.3\end{array}$ & $\begin{array}{l}68.8 \pm \\
22.5\end{array}$ & $\begin{array}{l}57.9 \pm \\
32.6\end{array}$ & $\begin{array}{l}65.4 \pm \\
30.2\end{array}$ & $\begin{array}{l}80.2 \pm \\
21.0\end{array}$ & $\begin{array}{l}15.4 \pm \\
47.1\end{array}$ \\
\hline$p$-value & 0.737 & 0.868 & 0.011 & 0.027 & 0.308 & 0.386 & 0.109 & 0.652 & .078 \\
\hline \multicolumn{10}{|c|}{ Preventive therapy types } \\
\hline Don't use & $\begin{array}{l}84.6 \pm \\
20.2\end{array}$ & $\begin{array}{l}84.1 \pm \\
16.2\end{array}$ & $\begin{array}{l}73.9 \pm \\
25.7\end{array}$ & $\begin{array}{l}53.4 \pm \\
44.5\end{array}$ & $\begin{array}{l}68.5 \pm \\
23.0\end{array}$ & $\begin{array}{l}57.0 \pm \\
32.7\end{array}$ & $\begin{array}{l}67.2 \pm \\
30.6\end{array}$ & $\begin{array}{l}80.4 \pm \\
21.0\end{array}$ & $\begin{array}{l}14.5 \pm \\
47.0\end{array}$ \\
\hline Co-trimoxazole & $\begin{array}{l}82.3 \pm \\
18.6\end{array}$ & $\begin{array}{l}81.8 \pm \\
9.9\end{array}$ & $\begin{array}{l}73.1 \pm \\
29.3\end{array}$ & $\begin{array}{l}41.8 \pm \\
44.4\end{array}$ & $\begin{array}{l}65.5 \pm \\
20.6\end{array}$ & $\begin{array}{l}56.2 \pm \\
33.4\end{array}$ & $\begin{array}{l}64.9 \pm \\
32.8\end{array}$ & $\begin{array}{l}80.7 \pm \\
20.9\end{array}$ & $\begin{array}{l}7.0 \pm \\
41.9\end{array}$ \\
\hline Isoniazid & $\begin{array}{l}79.2 \pm \\
24.8\end{array}$ & $\begin{array}{l}84.8 \pm \\
12.9\end{array}$ & $\begin{array}{l}64.5 \pm \\
27.3\end{array}$ & $\begin{array}{l}27.6 \pm \\
36.5\end{array}$ & $\begin{array}{l}69.9 \\
15.5\end{array}$ & $\begin{array}{l}62.5 \pm \\
29.0\end{array}$ & $\begin{array}{l}59.1 \pm \\
29.0\end{array}$ & $\begin{array}{l}77.0 \pm \\
20.1\end{array}$ & $\begin{array}{l}22.4 \pm \\
47.9\end{array}$ \\
\hline $\begin{array}{l}\text { Cotrimoxazole + } \\
\text { Isoniazid }\end{array}$ & $\begin{array}{l}89.3 \pm \\
18.6\end{array}$ & $\begin{array}{l}78.6 \pm \\
13.4\end{array}$ & $\begin{array}{l}50.7 \pm \\
31.7\end{array}$ & $\begin{array}{l}10.7 \pm \\
28.3\end{array}$ & $\begin{array}{l}66.1 \pm \\
18.3\end{array}$ & $\begin{array}{l}50.0 \pm \\
30.4\end{array}$ & $\begin{array}{l}51.4 \pm \\
24.9\end{array}$ & $\begin{array}{l}85.7 \pm \\
17.8\end{array}$ & $\begin{array}{l}7.1 \pm \\
35.9\end{array}$ \\
\hline$p$-value & 0.696 & 0.816 & 0.044 & 0.002 & 0.626 & 0.935 & 0.208 & 0.851 & 0.372 \\
\hline \multicolumn{10}{|c|}{ Current HAART regimen } \\
\hline
\end{tabular}




\begin{tabular}{|c|c|c|c|c|c|c|c|c|c|}
\hline Variables & OF* & LS* & $H W *$ & FW* & MW* & $\mathrm{HM}^{*}$ & DW* & PT* & SF* \\
\hline D4T-3TC-NVP & $\begin{array}{l}79.8 \pm \\
20.7\end{array}$ & $\begin{array}{l}82.8 \pm \\
11.8\end{array}$ & $\begin{array}{l}68.5 \pm \\
23.9\end{array}$ & $\begin{array}{l}47.0 \pm \\
44.9\end{array}$ & $\begin{array}{l}65.3 \pm \\
22.1\end{array}$ & $\begin{array}{l}55.4 \pm \\
34.9\end{array}$ & $\begin{array}{l}57.9 \pm \\
28.9\end{array}$ & $\begin{array}{l}79.0 \pm \\
22.8\end{array}$ & $\begin{array}{l}20.4 \pm \\
41.3\end{array}$ \\
\hline D4T-3TC-EFV & $\begin{array}{l}77.9 \pm \\
19.1\end{array}$ & $\begin{array}{l}78.1 \pm \\
13.0\end{array}$ & $\begin{array}{l}68.6 \pm \\
24.7\end{array}$ & $\begin{array}{l}26.5 \pm \\
39.1\end{array}$ & $\begin{array}{l}57.9 \pm \\
22.0\end{array}$ & $\begin{array}{l}51.5 \pm \\
35.1\end{array}$ & $\begin{array}{l}64.5 \pm \\
32.6\end{array}$ & $\begin{array}{l}73.5 \pm \\
15.7\end{array}$ & $\begin{array}{l}18.9 \pm \\
46.9\end{array}$ \\
\hline AZT-3TC-NVP & $\begin{array}{l}85.3 \pm \\
17.7\end{array}$ & $\begin{array}{l}81.9 \pm \\
10.7\end{array}$ & $\begin{array}{l}76.2 \pm \\
28.0\end{array}$ & $\begin{array}{l}39.1 \pm \\
41.4\end{array}$ & $\begin{array}{l}69.8 \pm \\
21.5\end{array}$ & $\begin{array}{l}54.3 \pm \\
30.6\end{array}$ & $\begin{array}{l}68.3 \pm \\
28.4\end{array}$ & $\begin{array}{l}81.6 \pm \\
21.4\end{array}$ & $\begin{array}{l}16.7 \pm \\
49.9\end{array}$ \\
\hline AZT-3TC-EFV & $\begin{array}{l}76.7 \pm \\
29.8\end{array}$ & $\begin{array}{l}90.2 \pm \\
35.8\end{array}$ & $\begin{array}{l}71.3 \pm \\
32.7\end{array}$ & $\begin{array}{l}45.3 \pm \\
47.9\end{array}$ & $\begin{array}{l}67.4 \pm \\
20.2\end{array}$ & $\begin{array}{l}51.4 \pm \\
29.3\end{array}$ & $\begin{array}{l}67.4 \pm \\
32.1\end{array}$ & $\begin{array}{l}81.9 \pm \\
21.7\end{array}$ & $\begin{array}{l}5.70 \pm \\
46.2\end{array}$ \\
\hline TDF-3TC-EFV & $\begin{array}{l}84.9 \pm \\
18.2\end{array}$ & $\begin{array}{l}83.7 \pm \\
11.3\end{array}$ & $\begin{array}{l}72.5 \pm \\
26.6\end{array}$ & $\begin{array}{l}52.3 \pm \\
45.1\end{array}$ & $\begin{array}{l}67.8 \pm \\
21.5\end{array}$ & $\begin{array}{l}58.6 \pm \\
32.2\end{array}$ & $\begin{array}{l}62.7 \pm \\
32.3\end{array}$ & $\begin{array}{l}81.9 \pm \\
19.1\end{array}$ & $\begin{array}{l}12.3 \pm \\
45.4\end{array}$ \\
\hline TDF-3TC-NVP & $\begin{array}{l}83.5 \pm \\
25.2\end{array}$ & $\begin{array}{l}83.2 \pm \\
11.4\end{array}$ & $\begin{array}{l}73.2 \pm \\
28.0\end{array}$ & $\begin{array}{l}47.5 \pm \\
48.5\end{array}$ & $\begin{array}{l}74.7 \pm \\
24.5\end{array}$ & $\begin{array}{l}51.8 \pm \\
32.9\end{array}$ & $\begin{array}{l}70.8 \pm \\
27.2\end{array}$ & $\begin{array}{l}83.82 \pm \\
1.1\end{array}$ & $\begin{array}{l}15.4 \pm \\
50.4\end{array}$ \\
\hline Others & $\begin{array}{l}82.8 \pm \\
17.3\end{array}$ & $\begin{array}{l}86.7 \pm \\
9.19\end{array}$ & $\begin{array}{l}73.3 \pm \\
30.5\end{array}$ & $\begin{array}{l}48.9 \pm \\
42.1\end{array}$ & $\begin{array}{l}68.5 \pm \\
23.3\end{array}$ & $\begin{array}{l}59.8 \pm \\
37.8\end{array}$ & $\begin{array}{l}64.8 \pm \\
28.9\end{array}$ & $\begin{array}{l}69.2 \pm \\
28.3\end{array}$ & $\begin{array}{l}9.09 \pm \\
46.3\end{array}$ \\
\hline$p$-value & 0.298 & 0.358 & 0.922 & 0.527 & 0.670 & 0.896 & 0.824 & 0.190 & 0.822 \\
\hline \multicolumn{10}{|l|}{ Comorbidity } \\
\hline Yes & $\begin{array}{l}82.5 \pm \\
21.6\end{array}$ & $\begin{array}{l}87.3 \pm \\
24.2\end{array}$ & $\begin{array}{l}19.9 \pm \\
4.98\end{array}$ & $\begin{array}{l}48.6 \pm \\
30.5\end{array}$ & $\begin{array}{l}69.8 \pm \\
23.3\end{array}$ & $\begin{array}{l}44.3 \pm \\
5.98\end{array}$ & $\begin{array}{l}59.2 \pm \\
32.7\end{array}$ & $\begin{array}{l}83.8 \pm \\
18.7\end{array}$ & $\begin{array}{l}5.60 \pm \\
41.8\end{array}$ \\
\hline No & $\begin{array}{l}84.3 \pm \\
19.5\end{array}$ & $\begin{array}{l}68.5 \pm \\
11.4\end{array}$ & $\begin{array}{l}19.3 \pm \\
5.49\end{array}$ & $\begin{array}{l}48.8 \pm \\
33.8\end{array}$ & $\begin{array}{l}67.6 \pm \\
21.8\end{array}$ & $\begin{array}{l}44.8 \pm \\
2.86\end{array}$ & $\begin{array}{l}64.7 \pm \\
30.5\end{array}$ & $\begin{array}{l}79.8 \pm \\
21.2\end{array}$ & $\begin{array}{l}14.1 \pm \\
46.3\end{array}$ \\
\hline$p$-value & 0.555 & 0.030 & 0.455 & 0.975 & 0.511 & 0.449 & 0.237 & 0.199 & 0.223 \\
\hline \multicolumn{10}{|c|}{ Opportunistic infections } \\
\hline Yes & $\begin{array}{l}83.3 \pm \\
20.3\end{array}$ & $\begin{array}{l}84.2 \pm \\
9.27\end{array}$ & $\begin{array}{l}18.7 \pm \\
5.86\end{array}$ & $\begin{array}{l}81.9 \pm \\
43.8\end{array}$ & $\begin{array}{l}66.2 \pm \\
23.9\end{array}$ & $\begin{array}{l}55.5 \pm \\
33.8\end{array}$ & $\begin{array}{l}62.8 \pm \\
33.6\end{array}$ & $\begin{array}{l}81.9 \pm \\
20.1\end{array}$ & $\begin{array}{l}11.1 \pm \\
46.6\end{array}$ \\
\hline No & $\begin{array}{l}84.1 \pm \\
19.8\end{array}$ & $\begin{array}{l}84.0 \pm \\
15.4\end{array}$ & $\begin{array}{l}19.5 \pm \\
5.31\end{array}$ & $\begin{array}{l}80.3 \pm \\
44.9\end{array}$ & $\begin{array}{l}68.3 \pm \\
21.7\end{array}$ & $\begin{array}{l}57.2 \pm \\
32.3\end{array}$ & $\begin{array}{l}63.8 \pm \\
30.5\end{array}$ & $\begin{array}{l}80.3 \pm \\
20.9\end{array}$ & $\begin{array}{l}12.9 \pm \\
45.5\end{array}$ \\
\hline$p$-value & 0.798 & 0.937 & 0.381 & 0.706 & 0.549 & 0.740 & 0.832 & 0.619 & 0.814 \\
\hline \multicolumn{10}{|c|}{ Adverse drug reactions } \\
\hline Yes & $\begin{array}{l}83.1 \pm \\
19.7\end{array}$ & $\begin{array}{l}86.2 \pm \\
23.2\end{array}$ & $\begin{array}{l}19.2 \pm \\
5.87\end{array}$ & $\begin{array}{l}50.6 \pm \\
46.2\end{array}$ & $\begin{array}{l}21.3 \pm \\
3.05\end{array}$ & $\begin{array}{l}53.7 \pm \\
34.7\end{array}$ & $\begin{array}{l}62.1 \pm \\
34.4\end{array}$ & $\begin{array}{l}79.0 \pm \\
21.1\end{array}$ & $\begin{array}{l}17.4 \pm \\
47.1\end{array}$ \\
\hline No & $\begin{array}{l}84.2 \pm \\
20.0\end{array}$ & $\begin{array}{l}83.5 \pm \\
11.7\end{array}$ & $\begin{array}{l}19.4 \pm \\
5.29\end{array}$ & $\begin{array}{l}48.4 \pm \\
44.3\end{array}$ & $\begin{array}{l}53.8 \pm \\
1.40\end{array}$ & $\begin{array}{l}57.7 \pm \\
32.0\end{array}$ & $\begin{array}{l}64.0 \pm \\
30.1\end{array}$ & $\begin{array}{l}80.9 \pm \\
20.7\end{array}$ & $\begin{array}{l}11.4 \pm \\
45.2\end{array}$ \\
\hline p-value & 0.702 & 0.217 & 0.725 & 0.737 & 0.21 & 0.404 & 0.666 & 0.540 & 0.379 \\
\hline \multicolumn{10}{|l|}{ Adherence status } \\
\hline Adherent & $\begin{array}{l}85.4 \pm \\
19.2\end{array}$ & $\begin{array}{l}83.3 \pm \\
11.2\end{array}$ & $\begin{array}{l}72.2 \pm \\
26.8\end{array}$ & $\begin{array}{l}50.1 \pm \\
43.7\end{array}$ & $\begin{array}{l}69.0 \pm \\
22.2\end{array}$ & $\begin{array}{l}58.2 \pm \\
32.1\end{array}$ & $\begin{array}{l}65.6 \pm \\
30.9\end{array}$ & $\begin{array}{l}79.6 \pm \\
21.0\end{array}$ & $\begin{array}{l}10.4 \pm \\
45.5\end{array}$ \\
\hline Non-adherent & $\begin{array}{l}80.8 \pm \\
21.1\end{array}$ & $\begin{array}{l}85.7 \pm \\
20.4\end{array}$ & $\begin{array}{l}71.2 \pm \\
27.7\end{array}$ & $\begin{array}{l}45.8 \pm \\
46.9\end{array}$ & $\begin{array}{l}60.7 \pm \\
21.6\end{array}$ & $\begin{array}{l}54.0 \pm \\
33.4\end{array}$ & $\begin{array}{l}59.1 \pm \\
30.7\end{array}$ & $\begin{array}{l}82.6 \pm \\
20.2\end{array}$ & $\begin{array}{l}17.9 \pm \\
45.5\end{array}$ \\
\hline$p$-value & 0.036 & 0.186 & 0.770 & 0.441 & 0.022 & 0.030 & 0.090 & 0.025 & 0.198 \\
\hline
\end{tabular}

\section{Discussion}

This study used HIV/AIDS-targeted instrument to assess the HRQoL and its associated factors among PLWHA in Addis Ababa, Ethiopia. The study showed that lower HRQoL mean scores were observed in sexual function, financial worries, and HIV mastery, while overall 
function, life satisfaction, and provider trust were the higher scores, respectively. Being female, older age, low household income, taking prophylactic therapy, low CD4 + count, and medication non-adherence were associated with lower mean quality of life scores.

Overall, PLWHA in the present study had moderate health status in majority of the domains. Our finding was in line with the previous study that sexual function of HIV patients was affected noticeably to a great extent [19]. This might be because PLWHA often avoid relationships, fear sexual intercourse, and being diagnosed with HIV by itself would change patient's sexual desire. ${ }^{28}$ Furthermore, people living with HIV infection might fear transmission of HIV and cultural hindrance to talk about the matter of sexuality with health professionals accounted for reduced sexual function [14]. On the other hand, similar to previous studies, PLWHA had good overall function [27, 28], however, higher than previous study conducted in a different setting in Ethiopia [29]. These high health state could indicate that patient's recieve appropriate patient care and counseling from healthcare providers, which led them less worry about their general health and subsequently had a better HRQoL. In addition, the study demonstrated that HIV mastery was lower than the study conducted by Dutra et al in Brazil [6]; indicating that HIV acceptance is a concern for patients living with HIV infection in Ethiopia. In contrary to other findings [30, 31], medication concern was lower in our study, but approximately in agreement with Brazilian finding [32]. These differences might be due to worries about the possible side effects and availability of interrupted medication supply within the facilities. These results demonstrated that health care providers should promote toward reducing stigmatization and discrimination of PLWHA, and provide pschosocial support to improve sexual function.

This study illustrated that sociodemographic characterstics notably affects the different HAT-QoL domains. Low household income was associated with high financial worries, in accordance with other findings that low income had a negative impact on patient HRQoL [27, 28, 33]. The fact that HIV infected patients with low financial status face insecurities and this could be a barrier to afford the basic expense [34]. Overall function, financial concern, sexual function, and HIV mastery were significantly lower in females than males; indicating that gender is negatively associated with an individual's HRQoL and these results were similar to other studies [30, 33]. In addition, women worry more about their health conditions than males, which is possibly associated with lower HRQoL [35]. On the other hand, a significant positive association between being married and health worries as well as sexual function was observed; this illustrates that patients might be discuss and solve sexual related problems with partner. The same study also revealed that higher domain scores were associated with a stable relationship [36]. The result demonstrated that focus should be given in reducing stigma and support the patients with pschosocial support as well.

Furthermore, the present study showed that health concern significantly decreased in patients older than 50 years. This could be due to deterioration of immunity with increasing age, in which expose patients to opportunity infections and worsen clinical outcomes; attribute for low health worries domain [28]. Our study demonstrated that educational status was associated with better HAT-QoL domains. This finding corroborate with previous studies done in Brazil [28, 32]. The improved HRQoL among educated patients might be attributed to their good understanding of the disease's long term complications, medication adverse effects and treatment outcomes [27]. On the other hand, as people live with HIV for more than 10 years, provider trust was increased while health worries decreased. This demonstrated that HIVinfected patients had a good physician-patient relationship that increase their understanding of the disease; improve HRQoL greatly [31].

Certain clinical characteratics were significantly associated with lower HAT-QoL domains. Participants who had a CD4 count < 200 cells/ $\mu \mathrm{L}$ and WHO stage IV reported significantly more health worries and medication worries. Likewise, Caliari JS. et al showed that there was a significantly negative association between CD4 count $<200$ cells/ $\mu \mathrm{L}$ and medication worries [30]. These results suggesting that patients who had low CD4 count were more acutely ill with a worsened immune system and this could be a concern for PLWHA and, in turn, may adversely affect HRQoL.

In the present study, medication adherence was significantly associated with medication concerns and provider trust domains. Our study found that $69.7 \%$ were adherent to HAART regimen, which is approximately similar to the study done in United State of America (68.0\%) [18], nonetheless higher than a study performed in Thailand (31.4\%) [37]. This evidences demonstrated that non-adherence to ART is also another major factor for compromised HRQoL among PLWHA [18, 31]. Patients negligence, fear of stigma, concern of disclosure of their HIV status through the use of such medications and differences in health education delivered by healthcare professionals might be attributed to non-adherence variations among studies in different settings [31].

The study has certain limitations. Since this study was cross-sectional study design, evaluation of HRQoL was not assessed possible variations over time. Secondly, as this study carried out in a single healthcare setting, the result might not represent the health status of HIV patients in Ethiopia. Despite all those limitations, our study has several strengths. First, we used a psychometrically robust and HIV/AIDS specific instrument to measure patient's HRQoL in Ethiopia; that would remarkable capture the different domains of HRQoL in PLWHA. In addition, the findings of the present study were also important in understanding the factors affecting the HRQoL of PLWHA. Secondly, clearly understanding predictors help clinicians to implement cost-effective interventional methods to improve HRQoL of the patients.

Page $12 / 15$ 
Finally, the study illustrates that measuring patient-perceived burden, could be used by policymaker to make evidence-informed decisionmaking in monitoring ART programs in the country.

\section{Conclusions}

Overall, HIV/AIDS patients in Addis Ababa had an average level of HRQoL. The most affected domains were sexual function, financial worries and HIV mastery while overall function, life satisfaction, and physician trust were better mean scores. Female gender, older age, household income, taking prophylactic therapy, low CD4 + count, and medication non-adherence strongly were associated with lower HRQoL. Therefore, healthcare providers and policymakers should be paid attention to medication adherence and psychosocial support to enhance HRQoL.

\section{Abberviations}

HAART: Highly active antiretroviral therapy, HAT-QoL: HIV/AIDS targeted quality of life, HRQoL: Health-related quality of life, PLWHA: People living with HIV/AIDS, WHO: World health organization.

\section{Declarations}

Ethics approval and informed consent: The study was approved by the Ethical Review Board of School of Pharmacy, College of Health Sciences, Addis Ababa University (Ref. No.: ERB/SOP/75/04/2019) and written informed consent was obtained from all patients.

Consent for publication: All authors have consented for publication.

Availability of data and material: The raw data are available upon request from the corresponding author.

Funding: No funding source

Conflict of interest: The authors declare that they have no competing interests.

\section{Acknowledgments}

We would like to extend our sincere gratitude to all patients who participated in this research as well as pharmacists, nurses, and physicians working in the hospital.

\section{Authors' contributions}

GTG and KYB conceived the research idea, did translation and transcultural adaptation of HAT-QoL questionnaire; GTG, GBG, KYB, YTW and AMF performed data analysis and interpretation; GTG and KYB write original draft of the manuscript;YTW, AMF and GBG: reviewed and edited the manuscript. All authors critically evaluated the final manuscript for important intellectual content and approved the final version of the manuscript.

\section{References}

1. Global UNAIDS, Statistics HIV. 2020. https://www.unaids.org/en/resources/fact-sheet. Accessed 02 September 2020.

2. Boyle BA. HIV in developing countries: a tragedy only starting to unfold. Aids Reader-New York. 2000;10(2):77-9.

3. UNAIDS. Fact sheet-latest statistics on the status of the AIDS epidemic; 2019. 2018. https://www.unaids.org/en/resources/fact-sheet . Accessed 02 September 2020.

4. Kibret GD, Ferede A, Leshargie CT, Wagnew F, Ketema DB, Alebel A. Trends and spatial distributions of HIV prevalence in Ethiopia. Infect Dis Poverty. 2019;8(1):2-9.

5. HAPCO. HIV prevention in Ethiopia National Road map; 2018. https://ethiopia.unfpa.org/en/hiv-prevention-ethiopia-national-road-map. Accessed 29 August 2020.

6. Dutra BS, Lédo AP, Lins-Kusterer L, Luz E, Prieto IR, Brites C. Changes health-related quality of life in HIV-infected patients following initiation of antiretroviral therapy: a longitudinal study. Brazilian J Infect Dis. 2019;23(4):211-7.

7. World Health Organization. Consolidated guidelines on the use of antiretroviral drugs for treating and preventing HIV infection: recommendations for a public health approach. World Health Organization; 2016. 
8. Chawla A, Wang C, Patton C, Murray M, Punekar Y, de Ruiter A, Steinhart C. A review of long-term toxicity of antiretroviral treatment regimens and implications for an aging population. Infectious diseases therapy. 2018;7(2):183-95.

9. George S, Bergin C, Clarke S, Courtney G, Codd MB. Health-related quality of life and associated factors in people with HIV: an Irish cohort study. Health Qual Life Outcomes. 2016;14(1):115.

10. Cooper V, Clatworthy J, Harding R, Whetham J, Brown A, Leon A, et al. Measuring quality of life among people living with HIV: A systematic review of reviews. Health Qual Life Outcomes. 2017;15(1):220.

11. Colombo GL, Di Matteo S, Antinori A, Medaglia M, Murachelli S, Rizzardini G. Economic evaluation of initial antiretroviral therapy for HIV-infected patients: an update of Italian guidelines. Clinicoecon Outcomes Res. 2013;5:489-96.

12. Loubiere S, Meiners C, Sloan C, Freedberg KA, Yazdanpanah Y. Economic evaluation of ARTs in resource-limited countries. Curr Opin Hiv Aids. 2010;5(3):225-31.

13. Hasanah $\mathrm{Cl}$, Zaliha AR, Mahiran M. Factors influencing the quality of life in patients with HIV in Malaysia. Qual Life Res. 2011;20(1):91-100.

14. Degroote S, Vogelaers D, Vandijck DM. What determines health-related quality of life among people living with HIV: an updated review of the literature. Archives of Public Health. 2014 Dec;72(1):1-10.

15. Mwesigire DM, Martin F, Seeley J, Katamba A. Relationship between CD4 count and quality of life over time among HIV patients in Uganda: a cohort study. Health Qual Life Outcomes. 2015;13(1):144.

16. Razera F, Ferreira J, Bonamigo RR. Factors associated with health-related quality-of-life in HIV-infected Brazilians. Int J STD AIDS. 2008;19(8):519-23.

17. Monteiro F, Canavarro MC, Pereira M. Factors associated with quality of life in middle-aged and older patients living with HIV. AIDS Care-Psychol Socio-Medical Asp AIDS/HIV. 2016;28:92-8.

18. Mannheimer SB, Matts J, Telzak E, Chesney M, Child C, Wu AW, et al. Quality of life in HIV-infected individuals receiving antiretroviral therapy is related to adherence. AIDS Care - Psychol Socio-Medical Asp AIDS/HIV. 2005;17(1):10-22.

19. Ferreira de Sousa JC, de Oliveira e Silva AC, Correia Paiva Leadebal OD, Queiroga Freitas FF, Silva Holmes E, Estrela de Albuquerque SG, et al. Quality Of Life Evaluation Of Elderly People Living With HIV/AIDS According To HAT-QOL. Int Arch Med. $2016 ; 1-10$.

20. Group TE. EuroQol-a new facility for the measurement of health-related quality of life. Health policy. 1990;16(3):199-208.

21. Ware JE, Sherbourne CD. The MOS 36-item short-form health survey (SF-36): conceptual framework and item selection. Med Care. 1992;30(6):473-83.

22. O'Connell K, Skevington S, Saxena S. Preliminary development of the World Health Organsiation's quality of life HIV instrument (WHOQOL-HIV). Soc Sci Med. 2003;57(7):1259-75.

23. Shahriar J, Delate T, Hays RD, Coons SJ. Commentary on using the SF-36 or MOS-HIV in studies of persons with HIV disease. Health Qual Life Outcomes. 2003;1(1):1-7.

24. Holmes WC, Shea JA. A New HIV/AIDS-Targeted Quality of Life (HAT-QoL) Instrument: Development, Reliability, and Validity. Med Care. 1998;36(2):138-54.

25. Surur AS, Teni FS, Wale W, Ayalew Y, Tesfaye B. Health related quality of life of HIV/AIDS patients on highly active anti-retroviral therapy at a university referral hospital in Ethiopia. BMC Health Serv Res. 2017;17(1):1-8.

26. Melaku T, Mamo G, Chelkeba L, Chanie T. Health-Related Quality of Life Among People Living with Human Immunodeficiency Virus on Highly Active Antiretroviral Therapy in Ethiopia: PROQOL-HIV Based Survey. Patient Related Outcome Measures. 2020;11:73.

27. Biambo AA, Adibe MO, Liman HM, Ukwe CV. Health-related quality of life of HIV-infected patients taking different antiretroviral regimens at a tertiary healthcare facility in northern Nigeria. Trop J Pharm Res. 2018;17(3):549-57.

28. Soares GB, Garbin CAS, Rovida TAS, Garbin AJÍ. Qualidade de vida de pessoas que vivem com HIV/AIDS assistidas no serviço especializado em Vitória (ES), Brasil. Cienc e Saude Coletiva. 2015;20(4):1075-84.

29. Vo QT, Hoffman S, Nash D, El-Sadr WM, Tymejczyk OA, Gadisa T, Melaku Z, Kulkarni SG, Remien RH, Elul B. Gender differences and psychosocial factors associated with quality of life among ART initiators in Oromia, Ethiopia. AIDS Behavior. 2016;20(8):1682-91.

30. Caliari J, de S, Reinato, Pio LAF, Lopes DPM, Reis LP, Gir RK. E. Quality of life of elderly people living with HIV/AIDS in outpatient followup. Rev Bras Enferm. 2018;71:513-22.

31. Oparah AC, Soni JS, Arinze HI, Chiazor IE. Patient-reported quality of life during antiretroviral therapy in a nigerian hospital. Value Heal Reg Issues. 2013;2(2):254-8.

32. Santos FD, Amaral LR, Nunes SF, Rosa CR, Milhomem Filho EO, Lima VA, dos Santos LN. Evaluation of Quality of Life in Women with HIV/AIDS According to the HAT-QoL. International Archives of Medicine. Int Arch Med. 2016;1-9.

Page $14 / 15$ 
33. Miyada S, Garbin AJI, Wakayama B, Saliba TA, Garbin CAS. Quality of life of people with HIV/AIDS - The influence of social determinants and disease-related factors. Rev Soc Bras Med Trop. 2019;52:0-1.

34. Galvão MT, Soares LL, Pedrosa SC, Fiuza ML, Lemos LD. Quality of life and adherence to antiretroviral medication in people with HIV. Acta Paulista de Enfermagem. 2015;28(1):48-53.

35. Medeiros RC, Medeiros JA, Silva TA, Andrade RD, Medeiros DC, Araújo JD, Oliveira AM, Costa MA, Cabral BG, Dantas PM. Quality of life, socioeconomic and clinical factors, and physical exercise in persons living with HIV/AIDS. Revista de saude publica. 2017;20;51:66.

36. Ming Z, Prybylski D, Cheng F, Airawanwat R, Zhu Q, Liu W, Huang S. Two-year prospective cohort study on quality of life outcomes among people living with HIV after initiation of antiretroviral therapy in Guangxi, China. J Assoc Nurse Aids C. 2014;25(6):603-13.

37. Li L, Lee SJ, Wen Y, Lin C, Wan D, Jiraphongsa C. Antiretroviral therapy adherence among patients living with HIV/AIDS in Thailand. Nurs Health Sci. 2010;12(2):212-20.

\section{Figures}

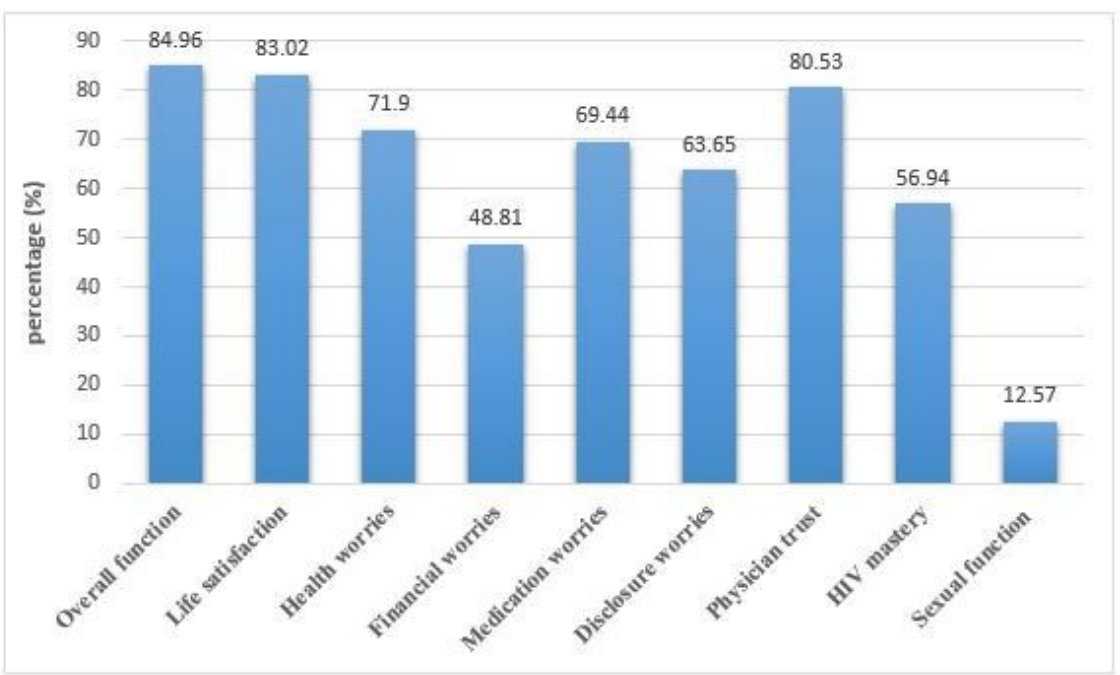

Figure 1

HAT-QoL domains mean scores among people living with HIV/AIDS and HAART

\section{Supplementary Files}

This is a list of supplementary files associated with this preprint. Click to download.

- Supplementaryfile1.docx 